\title{
A New Approach on Digital Blood Pressure Measurement Method for u-Healthcare Systems
}

\author{
Boyeon $\mathrm{Kim}^{1}$, Wenhai $\mathrm{Jin}^{2}$, Sung Hoon $\mathrm{Woo}^{3}$ and Yunseok Chang ${ }^{2}$ \\ ${ }^{1}$ Division of Computer Software and Engineering, Hanyang University, Seoul, Korea \\ ${ }^{2}$ Department of Computer Engineering, Daejin University, Pocheon, Korea \\ ${ }^{3}$ Department of Research, uMedix Co, Sungnam, Korea \\ cosmos@daejin.ac.kr
}

\begin{abstract}
The traditional blood pressure measurement method so called Kortokoff method has to apply strong air pressure on patient's artery that could give severe damage on artery, and has the problem that method cannot measure the blood pressure in a series or continuously. But recent engineering technology can give new solutions that can solve the problems by using digital sensors and microcomputer. In this paper, we proposed a kind of non-Kortokoff method that can get the blood pressure from patient's digitalized arterial pulse waveform analysis without air pressure on patient's artery. This method can check patient's blood pressure in a series through converting the continuous arterial pulse waveform into the absolute systolic and diastolic values with pre-defined blood pressure mapping equation from experiments. The experimental results showed that the proposed non-Kortokoff blood pressure measurement method has very good accuracy over $95 \%$ compare to commercial tonometers in continuous measurement. Also, the proposed non-Kortokoff blood pressure measurement method can be designed and implemented as a small device since it has no mechanical air pressure compartment, and applied on the u-Healthcare service areas very effectively such as a digital blood pressure checker for usual life blood pressure monitoring along with the medical device for home and hospitals.
\end{abstract}

Keywords: Arterial pulse waveform, Non-Kortokoff method, Continuous blood pressure measurement, $u$-Healthcare

\section{Introduction}

In modern society, mobile devices and wireless internet become strong utilities for data communication and multimedia applications. These utilities also become a kind of complex information technology such as smartphones and smart devices, and turn the traditional methodologies into the ubiquitous environment. The u-Healthcare area is one of the most useful application areas of the smart device on management of one's health status and information such as blood pressure, body temperature, heart rate, body composition and so on. The tonometer is one of the most important medical devices from home to hospital. But existing tonometer has drawback that it has to apply high pressure on the artery and it can give one's blood pressure no more than 5 consecutive checks. Thus the existing tonometer cannot be applied on 24-hours continuous blood pressure measurement for hypertension patient in usual life. The traditional method which known as Kortokoff method measures patient's blood pressure by using air pressure cuffs and stethoscope sound [1]. Today's doctors still check patient's blood pressure with this method. According to the development of information technologies, automatic electronics tonometer is widely used in home and 
hospitals since it is handy and has lots of usability. As it adopts oscillometric method, a sort of advanced Kortokoff method, and also has the same problem that it has fatal step to stop the artery completely as like the traditional tonometer. If the step repeats over and over again, the artery would be damaged seriously and that's why the Kortokoff method cannot be applied in series or continuous measurement. Especially, this method could make severe damage to a hypertensive needs very intensive usual blood pressure check [2]. To solve this problem, an absolute new non-Kortokoff method has to be developed that can measure the blood pressure without blocking artery. Although Osamu and his fellows had developed the 24hours continuous measuring tonometer in Japan from 2005, still it bases on the Kortokoff method and could not apply over 5 times [3-4]. In this paper we proposes a new concept called nonKortokoff method that can apply on 24-hours continuous blood pressure measurement, and verified its effectiveness though experiments with digital pressure sensor and smartphone. This work could give some important engineering bases to develop a next generation digital tonometer, and enhance the continuous measurement ability of existing tonometer.

\section{Related Works}

According to the growth of the ubiquitous environment, various u-Healthcare solutions are being developed to provide the related services. Most of these services provide medical healthcare information for inspection and treatment with wireless networks and digital signal processing techniques over the existing medical devices [5]. Zigbee wireless network based biomedical signal transmission system is one of the hottest research areas in ubiquitous health care [6-7], and many researches about the BAN (Body Area Network) based bio-information system has also being performed by using near field communication network on biomedical signal processing area [8]. Although these works and systems has high-end electronics sensor devices to get the various bio signals, the blood pressure still can be measured through the Kortokoff method or advanced Kortokoff method so called oscillometric method. In recent works, some blood pressure estimation methods with ECG/PPG signals had proposed that has not enough accuracy and stability and not accepted as a new blood pressure measurement method yet [9-10]. Other researches shows the mechanical artery variation can help the blood pressure estimation, and are still the same kind of existing tonometer since they are also a sort of Kortokoff method [11-13]. Several recent works focused on minimizing external pressure on artery and proposes various innovative blood pressure measurement algorithms [14]. The non-Kortokoff method proposed in this paper can estimate the blood pressure with the information resulted from arterial pulse waveform analysis and could be the most innovative method.

\section{Methodology}

In This work, we propose a new blood pressure measurement method for 24-hours continuous monitoring. It is a kind of the non-Kortokoff method that need not apply the high pressure on patient's artery or vain, and can be easily implemented with several digital electronics parts such as absolute air pressure sensor unit, AC/DC amplifier with ADC converter, microcomputer and application program. If a digital sensor that can produce the absolute air pressure value is located on the skin over an artery, the absolute pressure from the skin contacted part can be measured. From this pressure value, we can calculate the absolute arterial blood pressure through monitoring the pressure variation of the artery as follows. 


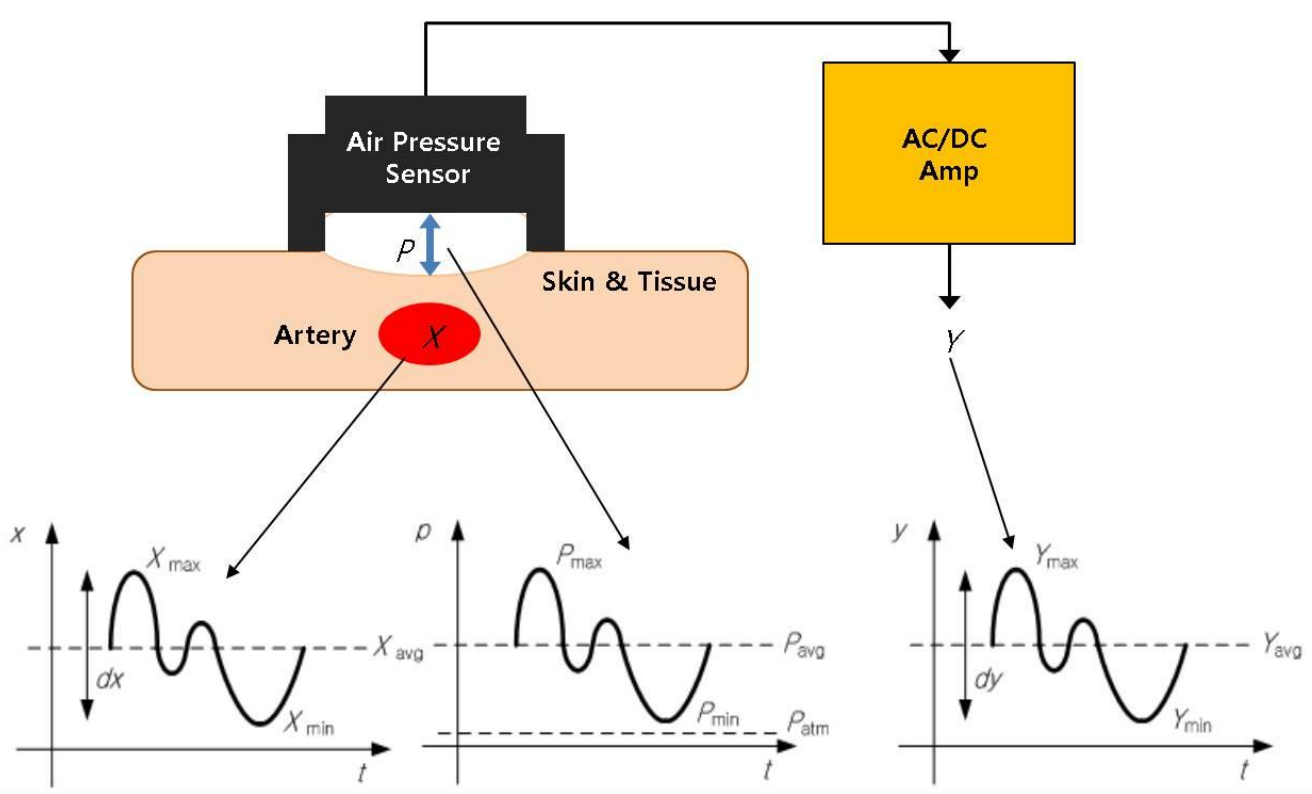

Figure 1. Basic Concept of Non-Kortokoff Measurement Method

In Figure $1, P_{a t m}$ represents the pressure of current atmosphere, $X$ shows the real blood pressure variation, $Y$ shows the digital output values from the AC/DC amplifier and $P$ shows the pressure measured between air pressure sensor and skin. The $d x, d y$ means the variation of the $X$ and $Y$ values between $X_{\max }$ and $X_{\min }, Y_{\max }$ and $Y_{\min }$ respectively. $P_{\text {avg }}, X_{\text {avg }}$ and $Y_{\text {avg }}$ are also the average value of $P, X$ and $Y$ values.

Since the digital sensor output can only be collected as a series of digital values through ADC converter in AC/DC amplifier, we can only get the $Y$ values related to $X$ values. Therefore, we have to find out the mapping relation $Y$ to $X$ as a sort of transition function. If we can find the mapping relation between $P_{\text {avg }}$ and $X_{\text {avg }}$ along with $d y$ and $d x$ through many experimental results, we can calculate the exact $X_{\text {avg }}$ and $d x$. The systolic and diastolic can be calculated from the $X_{\text {avg }}$ with $d x$. To get the mapping relation, we draw a graph $P$ vs. $d y / d x$ as shown in Figure 2 with relation equation (1).

$$
P(\Delta P)=a(d y / d x)+b
$$

If we can get $X_{\text {avg }}$ and dx with the existing tonometer from many volunteers with various $\Delta P \mathrm{~s}$ and $d y \mathrm{~s}$, we can get angle $a$, and offset $b$ through regression that $a$ can be the specific angle of a person. Therefore we can make a blood pressure mapping equation (2) for $X_{\text {avg }}$ along with the angle $a$, and can find out $\alpha$ and $\beta$ through regression from various $X_{\text {avg }}$ and $a$ values.

$$
X_{\text {avg }}=\alpha(a)+\beta
$$

Now we can conclude another blood pressure mapping equation (3) and can calculate the systolic and diastolic from the $d y$ under constant $P . P_{\text {avg }}$ and $d x$ can be checked at any point during measurement, we can easily calculate the systolic and diastolic values at any time and continuously.

$$
\begin{aligned}
& d x=d y /((P-b) / a) \\
& \text { Systolic }=X_{a v g}+d x \\
& \text { Diastolic }=X_{a v g}-d x
\end{aligned}
$$




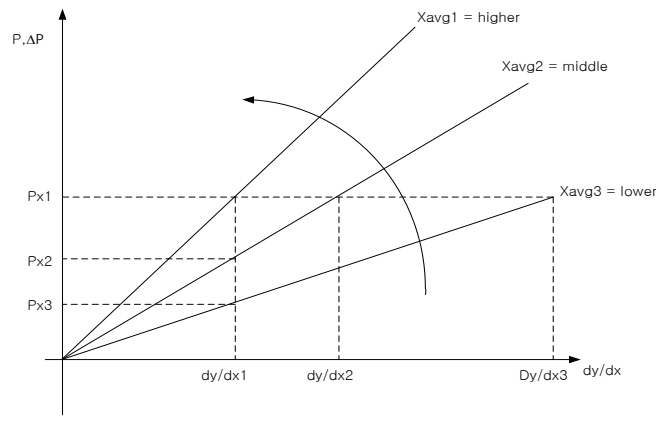

Figure 2. Relation between $P$ and $d y / d x$ for $X_{\text {avg }}$

This method need not block or stop the arterial blood current to get the $P$ and the $d y$ values. The digital air pressure sensor located on the skin just measures the $P$ and $d y$ continuously. So, if the blood current blocked, $d y$ would be dropped to 0 , then this method is unable to measure the blood pressure and it can be distinguished from the existing Kortokoff type or oscillometric type blood pressure measurement method.

\section{Measurement Environment}

\subsection{Design of Non-Kortokoff Type Tonometer}

In this work, we designed and implemented a sensor unit to test the proposed method. This non-Kortokoff type sensor unit can be connected with the android smartphone through the Bluetooth link as shown in Figure 3. We also implement an android app that can measure the blood pressure including systolic, diastolic and pulse by using the pressure parameters and arterial pulse waveforms from the sensor unit.

The sensor unit has air membrane digital pressure sensor that can out the absolute pressure value, CSR BC4-ext MPU circuit and wrist-banded enclosure. This sensor unit works only just the air membrane lies on the wrist artery instead of air pressing mechanism of the existing tonometer. The digital pressure sensor sends the arterial pulse variation data to the digital input port of the MPU through the $\mathrm{I}^{2} \mathrm{C}$ interface. The MPU sends the data to the paired smartphone through the Bluetooth link.
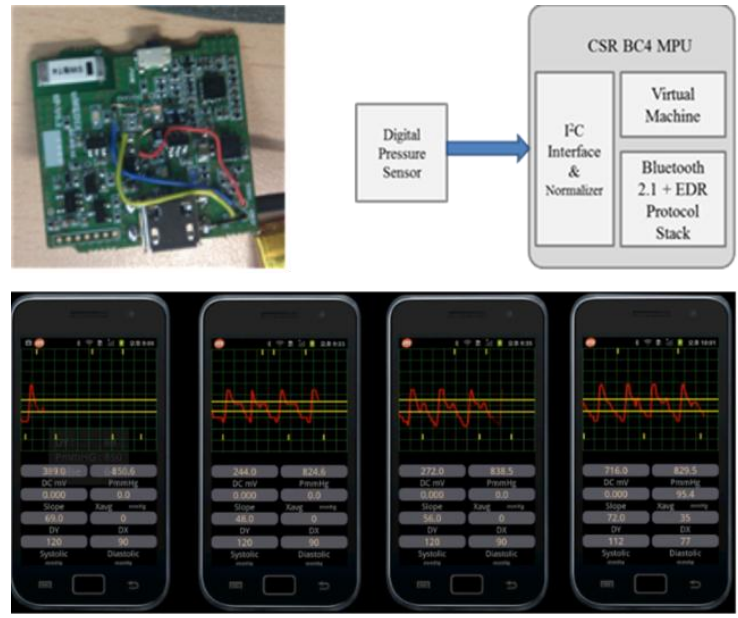

Figure 3. Non-Kortokoff Type Sensor Unit and Smartphone App 


\subsection{Experimental Environments and Procedures}

For the reasoning of the proposed method, 164 volunteers joined in the experiment over 2 rounds. At first round, a volunteer tried existing wrist-type tonometer and non-Kortokoff sensor unit 5 times alternatively, and tried again at least after a week from the previous round. We used Omron, one of the most popular commercial products as the existing wrist-type tonometer. Table 1show the experiment parameters applied on the experiments. Total 6 data excluding the highest and lowest data for each round are applied to get the average results for a victim. The experiments are performed during 1 minute for a trial. The arterial pulse waveforms of all trials had been recorded and analyzed the blood pressure data through afterwork from video.

Table 1. Experiment Parameters

\begin{tabular}{l|c}
\hline \multicolumn{1}{c|}{ Parameters } & Value(s) \\
\hline Number of Volunteers & 164 \\
Trials / Rounds per victim & $5 / 2$ \\
Measuring elaspe time & $60 \mathrm{sec}$ \\
Atmosphere pressure & $756 \mp 5 \mathrm{mmHg}$ \\
Sampling rate & $120 \mathrm{~Hz}$ \\
ADC Resolution & 20 bits (8bits down sampling) \\
Bluetooth & $2.1+$ EDR \\
\hline
\end{tabular}

\section{Experimental Results}

\subsection{Blood Pressure Mapping Equation}

If we want to find one's blood pressure value, we have to find out the coefficients $a, b, \alpha$ and $\beta$ of the relation equaton (1) and mapping equation (2) as mentioned in chapter 3 . From many experimental results, we have got these coefficients through transition functions in Fig. 4 with MatLab regression module. Figure 4a shows the transition function of the relation between Pressure varient angle $P_{\text {ang }}$ and $d y$ which is needed to calculate the transition function Figure $4 \mathrm{~b}$ that shows the transition function of angle $a$ and $d y / d x$ of relation equation (1).

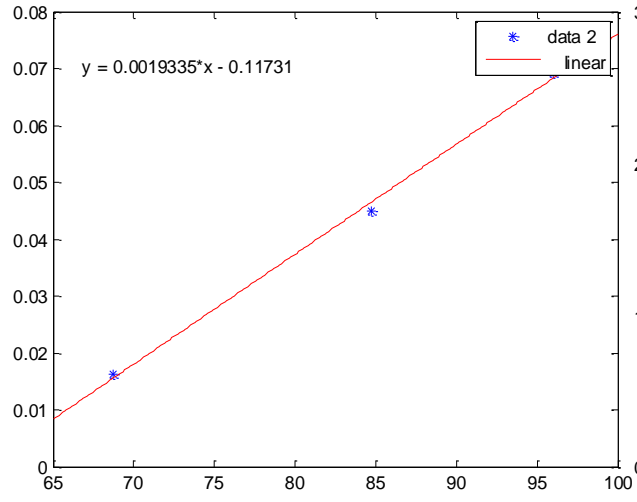

a) $P_{\text {ang vs }} d y$

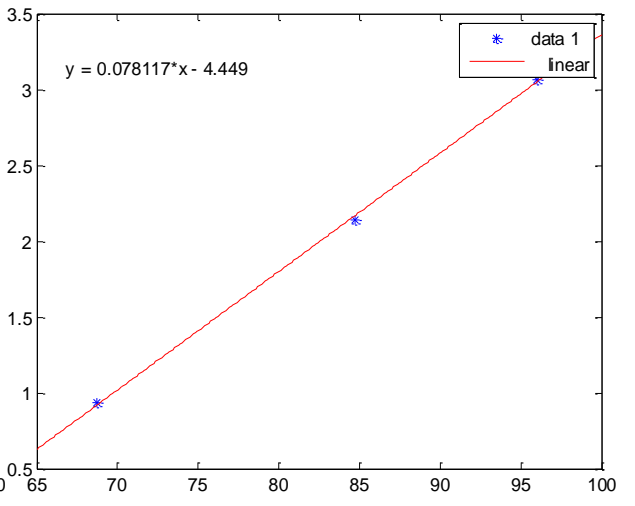

b) $a$ vs $d y / d x$

Figure 4. Transition Function for Blood Pressure Equation 


\subsection{Type of Arterial Pulse Waveform}

During experiment, we found that an arterial pulse waveform can be varied according to the victim's blood pressure characteristic such as hypertension, normal and hypotension as shown in Figure 5. The main difference among the characteristics lies on the shape of the arterial pulse waveforms, not on the amplitude of the waveforms, and the PQRS points those characterize the heartbeat pressure variant curve in artery and vain. Therefore, we have to measure the victims' basic blood pressure with Omron and grouped them in three blood pressure characteristics before each experiment. For the convenience of the experiment, we grouped a victim who has over $140 \mathrm{mmHG}$ systolic into the hypertension, under $90 \mathrm{mmHG}$ systolic was grouped into the hypotension, and between 140 and $90 \mathrm{mmHG}$ systolic was grouped into the normal.

As like the blood pressure characteristics, the blood pressure mapping equation and relation equation also have to match their coefficients according to the patient's blood pressure characteristics since each characteristics has large gaps among the arterial pulse waveforms and these gaps would make much difference on coefficient of the equations. Therefore, we calculated the coefficients in three groups along with the blood pressure characteristics from the results of Omron, and applied the arterial pulse waveform input data according to their own group.

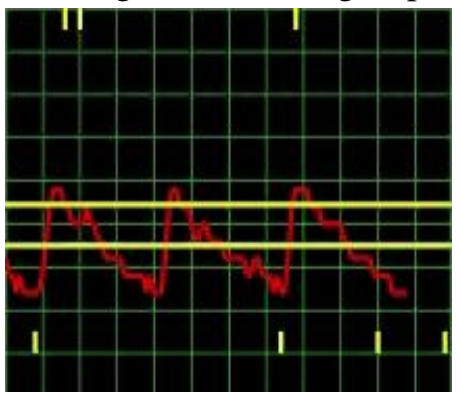

a) Hypertension

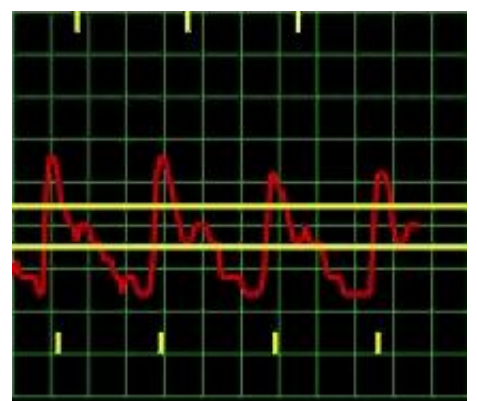

b) Normal

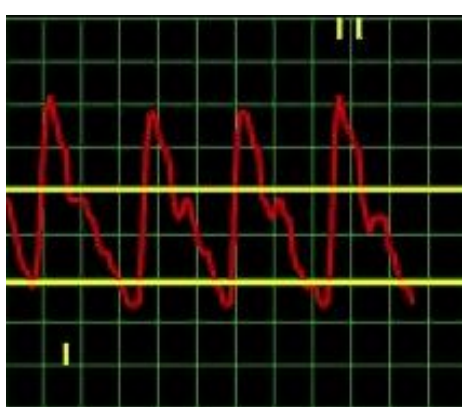

c) Hypotension

Figure 5. Types of Arterial Pulse Waveforms

\subsection{Accuracy Comparison Results}

Table 2 shows the average accuracy of the experimental results of systolic, diastolic and pulse compares to the basic blood pressures for every volunteers of each group. The basic blood pressure means a volunteer's average systolic, diastolic and pulse with Omron measured at every experiment. The accuracy shows an average puncture of the results compares to the average basic blood pressure on all trials of a volunteer. Since the blood pressure mapping equation can produce the effective systolic and diastolic values only at the case of catching the arterial pulse correctly. Therefore, at each trial, the missed cases those have somewhat large or less pulse error over $25 \%$ of volunteer's averge pulse are eliminated because it would be a kind of incorrect arterial pulse catch. For all trials of volunteers, the missed cases reached $12.8 \%$ for hypertentios, $7.3 \%$ for normal and $26.3 \%$ for hypotension.

Table 2. Accuracy Comparison Results

\begin{tabular}{|c|c|c|c|c|}
\hline \multirow{2}{*}{ Group } & \multicolumn{4}{|c|}{ Accuracy (\%) } \\
\cline { 2 - 5 } & Volunteers & Systolic & Diastolic & Pulse \\
\hline Hypertension & 48 & 95.4 & 92.4 & 91.4 \\
\hline
\end{tabular}




\begin{tabular}{|c|c|c|c|c|}
\hline \multirow{2}{*}{ Group } & \multicolumn{4}{|c|}{ Accuracy (\%) } \\
\cline { 2 - 5 } & Volunteers & Systolic & Diastolic & Pulse \\
\hline Normal & 94 & 95.7 & 93.6 & 96.7 \\
\hline Hypotension & 32 & 93.2 & 90.8 & 94.8 \\
\hline Average & & $\mathbf{9 5 . 2}$ & $\mathbf{9 2 . 2}$ & $\mathbf{9 6 . 0}$ \\
\hline
\end{tabular}

The results show the accuracy has more than $95 \%$ for systolic, $93 \%$ diastolic and $96 \%$ for pulse. It also show that the proposed method has highly compliant with the Omron for systolic of hypertension and normal group. But the proposed method has weak correctness for hypotension group and espicially for diastolic. It would be resulted from the nonlinear output response characteristics of the digital sensor unit with the pressure applied. But Omron also has high error rate for hypotension group and diastolic, low systolic accuracy gap could show the high correctness and reliability of the proposed method. From the results of Table 2, the proposed method can measure the blood pressure less than 5\% accuracy gap compare with the Omron.

\section{Conclusion}

The traditional blood pressure measurement method so called Kortokoff method has severe drawback in case of continuous measurement caused by applying high pressure on artery. But the proposed non-Kortokoff method can overcome the problems of the existing tonometer, and showed its effectiveness through the experiment using digital sensor unit and smartphone instead of air-pressure mechanical device. The new method can calculate systolic and diastolic directly from the arterial pulse waveform according to the blood pressure characteristics, and the experimental results showed that the proposed method could have nice accuracy more than $95 \%$ error rate for systolic without any step that blocks the artery completely as like the traditional method.

These results can prove the proposed method not only can replace the existing method but also can be effectively applied on the areas of usual or 24-hours continuous blood pressure measurement. In fact, many trials of high pressured blood pressure measurement could give severe damage to hypertension patients who really need to check the blood pressure in series or continuously in usual life. Therefore, the proposed method can provide a new engineering principle for hypertension patient. It also can be implemented in a small and lightweight device that can be connected to the mobile device or smartphone for 24-hours continuous checking under ubiquitous environment comes to the next generation ubiquitous digital tonometer that can solve the problem of the existing tonometer. This method can be effectively applied on the u-Healthcare service area for ubiquitous environment and can provide an innovative basis of the continuous blood pressure measurement more than ever existing methods.

\section{References}

[1] B. Williams, "Achieving blood pressure control in clinical practice", Journal of the American Society of Hypertension, vol. 2, no. 4, (2008).

[2] G. Ray, J. Nawarskas and J. Anderson, "Blood pressure monitoring technique impacts hypertension treatment”, Journal of General Internal Medicine, vol. 27 no. 6, (2012), pp. 6323-629. 
[3] G. Yasuda, N. Ogawa, G. Shimura, D. Ando, K. Shibata, S. Umemura and O. Tochikubo, "Effects of perindopril on 24-h blood pressure in hypertensive patients with diabetic nephropathy", American Journal of Hypertension, vol. 17, no. 5, (2004).

[4] O. Tochikubo, Y. Kaneko, Y. Yukinari and I. Takeda, "Noninvasive measurement of baroreflex sensitivity index using an indirect and continuous blood-pressure recorder," Japanese Heart Journal, vol. 27 no. 6, (2008), pp. 849-857.

[5] W. Ryu, E. Kim, K. An, S. Woo and Y. Chang, "A bluetooth based 5-HD measurement system for uHealthcare," International Journal of Control and Automation, vol. 6, no. 1, (2013), pp. 141-150.

[6] Y. Cha and G. Yoon, "Ubiquitous health monitoring system for multiple users using a zigbee and WLAN uual-network," telemedicine and e-Health, vol. 15, no. 9, (2009), pp. 891-897.

[7] Y. Chang and B. Kim, "A wireless ECG measurement system based on zigbee USN," The Korea Information Processing Society Transactions: Part C, vol. 18-C, no. 3, (2011), pp. 195-198.

[8] C. Otto, A. Milenkovic, C. Sanders and E. Jovanov, "System architecture of a wireless body area sensor network for ubiquitous health monitoring," Journal of Mobile Multimedia, vol. 1, (2006), pp. 307-326.

[9] Y. Yoon, J. and G. Yoon, "Non-constrained blood pressure monitoring using ECG and PPG for personal healthcare," Journal of Medical Systems, vol. 33, (2009), pp. 261-266.

[10] W. Shin, Y. Cha and G. Yoon, "ECG/PPG integer signal processing for a ubiquitous health monitoring system”, Journal of Medical Systems, vol. 34, (2010), pp. 891-898.

[11] D. Nair, S-Y Tan, H-W Gan, S-F Lim, J. Tan, M. Zhu, H. Gao, N-H Chua, W-L Peh and K-H Mak, "The use of ambulatory tonometric radial arterial wave capture to measure ambulatory blood pressure: the validation of a novel wrist-bound device in adults," Journal of Human Hypertension advance online publication, (2007).

[12] M. Ahn, J. Choi, I. Son, S. Lee and K. Kim, "Estimated blood pressure algorithm of wrist wearable pulsimeter using by hall device," Journal of the Korean Magnetics Society, vol. 20, no. 3, (2010).

[13] J. Kang, T. Yoo and H. Kim, "A wrist-worn integrated health monitoring instrument with a telereporting device for telemedicine and telecare," IEEE Transactions on Instrument and Measurement, vol. 55, no. 5, (2006).

[14] K. Barbe, W. Van Moer, "An innovative oscillometric blood pressure measurement: Getting rid of the traditional envelope," Proceedings of the 2012 Medical Measurements and Applications (MeMeA), IEEE Conference Publications, (2012).

\section{Authors}

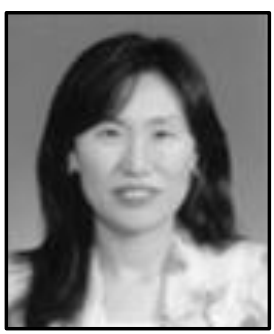

Boyeon Kim, she received her B.S degree in Computer Science from Ewha Woman's University 1989, M.S degree in Computer Engineering and Ph.D. degree in Biomedical Engineering from Seoul National University, 1991 and 1998 respectively. From 2000 to 2006, she had worked as an assistant professor in department of Electrical and Electronics and Computer Communications. She is currently working as a research professor in division of Computer Software and Engineering, Hanyang University. Her main interests has been reached on Biomedical engineering, Bio signal processing, Biomedical information, Artificial intelligence, Decision making systems and u-Healthcare information systems

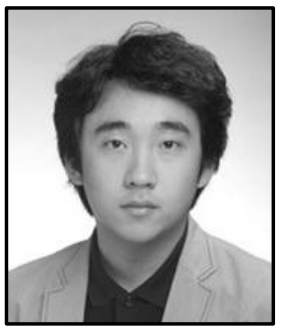

\section{Wenhai Jin}

He received B.S degree in Computer Engineering, and currently studies on the smart device and app programming in the graduate school in Daejin University. He had been joined on several research projects on biomedical information system and implemented various applications for iOS and android. His main research areas are Micro-embedded systems, Micro robots, Smartphone applications and Bio information systems. 


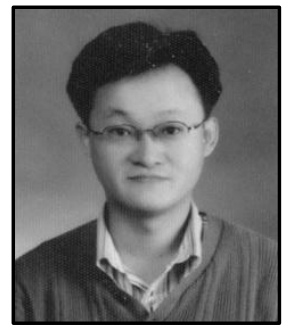

Sung Hun Woo, he is currently working as a CEO of uMedix Co., Korea from 2010. He received Ph.D. degree in Electronics at Korea Advanced Institute of Science and Technology (KAIST) at 2001. He worked as a chief researcher at IC team in Hynix Semiconductor at 2003 and now focuses on the ubiquitous healthcare system, devices and services at Korea and China. His main research areas are High definition video, Digital signal processing, u-Healthcare device and its services.

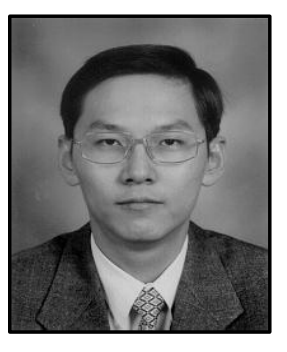

Yunseok Chang, he received his B.S. degree in Physics 1988, M.S. and $\mathrm{Ph} . \mathrm{D}$. in Computer Engineering from Seoul National University 1990 and 1998. He researched distributed parallel computing and data clustering at Department of EE-System, University of Southern California from 2000 to 2001 as a visiting scholar. He is currently working as a professor at the department of Computer Engineering, Daejin University, Korea. His research areas are concentrated on the Fundamental computer system design, RAID storage, Micro embedded system, Mobile device programming, u-Healthcare device and its services. 
International Journal of Bio-Science and Bio-Technology Vol.7, No.1 (2015) 